\title{
A Comparison of the Electron Density Variations in the F2 Region at Kokubunji and Yamagawa during the Night
}

\author{
By T. YONEZAWA
}

Central Radio Wave Observatory

\begin{abstract}
By comparing the electron density variations in the $\mathrm{F} 2$ region during the night at Kokubunji and Yamagawa it has been made clear that, if we assume a linear law of electron disappearance and somewhat higher temperature of the upper atmosphere at Yamagawa than at Kokubunji, the observational data fit theoretical expectation rather well, but a quadratic law can hardly be reconciled with the observations, unless recombination coefficient changes markediy with height.
\end{abstract}

\section{Introduction}

In a previous report ${ }^{(1)}$ we made an analysis of the electron density variations during the night at Kokubunji and at Washington and revealed that electrons disappear more probably according to a linear law rather than a quadratic one. We have now made a further study along the same line by comparing the electron density variations in the $\mathrm{F} 2$ region during the night at two sites separated not very far from each other, namely at Kokubunji $\left(35^{\circ} 42.4^{\prime} \mathrm{N}, 139^{\circ} 29.3^{\prime} \mathrm{E}\right)$ and at Yamagawa . $\left(31^{\circ} 12.5^{\prime} \mathrm{N}, 130^{\circ} 37.7^{\prime} \mathrm{E}\right)$, and the conclusion of the previous paper has again been confirmed. As a by-product we have also obtained a result that the upper atmospheric temperature above Yamagawa is probably somewhat higher than that above Kokubunji. 2. Method

The electron density variation during the night is governed by the following equation, if we adopt the linear law of electron disappearance :

$$
\frac{1}{N} \frac{d N}{d t}=-B_{0} e^{-\frac{z_{m}-z_{0}}{H}}-\frac{1}{T} \frac{d T}{d t}
$$

where $N$ is the electron density, $t$ is the time, $B_{0}$ is the attachment coefficient multiplied by the density, at a standard height $z_{0}$, of atoms and molecules to which electrons attach themselves, $z_{m}$ is the height of the level considered, $H$ is the local scale height and $T$ is the absolute temperature. In the following we shall take the maximum electron density of the $\mathrm{F} 2$ region as $N$ and $h_{p}$ as $z_{m}$. Writing down Eq. (1) for Kokubunji and Yamagawa and taking the differences of the both sides, we obtain

$$
\frac{d}{d t} \ln \frac{N_{2}}{N_{1}}=-B_{0}\left(e^{-\frac{z_{m 2}-z_{0}}{H_{2}}}-e^{-\frac{z_{m 1}-z_{0}}{H_{1}}}\right)+\text { const. }
$$




$$
\simeq B_{0}\left(\frac{z_{m 2}}{H_{2}}-\frac{z_{m 1}}{H_{1}}\right)-B_{0} z_{0}\left(\frac{1}{H_{2}}-\frac{1}{H_{1}}\right)+\text { const., }
$$

where we have assumed that the relative temperature variation is the same at both sites (in this case, const. $=0$ ) or the temperature decrease is exponential, and where the letters with subscripts 1 and 2 denote the quantities at Kokubunji and Yamagawa, respectively; $B_{0}$ is also assumed to be the same at the both stations.

Now, if the difference between $H_{1}$ and $H_{2}$ is not large compared with $H_{1}$ and $H_{2}$ themselves we can replace $H_{1}$ and $H_{2}$ with their average value $\bar{H}$ in Eq. (2) to a very rough approximation and obtain

$$
\begin{aligned}
\frac{d}{d t} \Delta \ln N & \simeq \frac{B_{0}}{\bar{H}} \Delta z_{u}+\text { const., } \\
\Delta z_{m} & \equiv z_{m 2}-z_{m 1}, \\
\Delta \ln N & \equiv \ln N_{2}-\ln N_{1} .
\end{aligned}
$$

Eq. (3) shows that there should be a linear relation between $\frac{d}{d t} \Delta \ln N$ and $\Delta z_{m}$, if time variation of $\bar{H}$ (or $H_{1}$ and $H_{2}$ ) is neglected. This is roughly the case as will be described in the next section, which seems to indicate that the method of analysis here adopted is not very unreasonable.

On the other hand, if electrons disappear by recombination, we shall be led to the following equation by a similar analysis :

$$
\begin{aligned}
\frac{d}{d t} \Delta \ln N & \simeq-\alpha . \Delta N+\text { const., } \\
\Delta N & \equiv N_{2}-N_{1},
\end{aligned}
$$

where $\alpha$ is the recombination coefficient which is assumed not to change markedly with height, as may be expected on theoretical grounds. But Eq. (6) can not be verified by observation (cf. $\S 3$ ),

Having thus established the approximate correctness of our analysis, we have then fitted Eq. (2) to observational data. If time variations of $H_{1}$ and $H_{2}$ are neglected as before, Eq. (2) becomes

$$
\frac{d}{d t} \Delta \ln N \simeq \frac{\dot{B}_{0}}{H_{2}} z_{m 2}-\frac{B_{0}}{H_{1}} z_{m 1}+\text { const. }
$$

Using the observational data of $\Delta \ln N, z_{m 2}$ and $z_{m 1}$, we can determine the most probable values of parameters $B_{0} / H_{1}$ and $B_{0} / H_{2}$ by the method of least squares, and from these we can obtain the ratio between the absolute temperatures of the upper atmosphere above Kokubunji and Yamagawa, for the scale height $H$ is proportional to the absolute temperature.

3. Results and discussions

Using median values of observational data for respective months we have calculated the values of $\frac{d}{d t} \Delta \ln N$ by numerical differentiation and plotted them (abscissa) against $\Delta h_{p}$ (ordinate) in Fig. 1, (a)-(f) for some months in 1949. The 


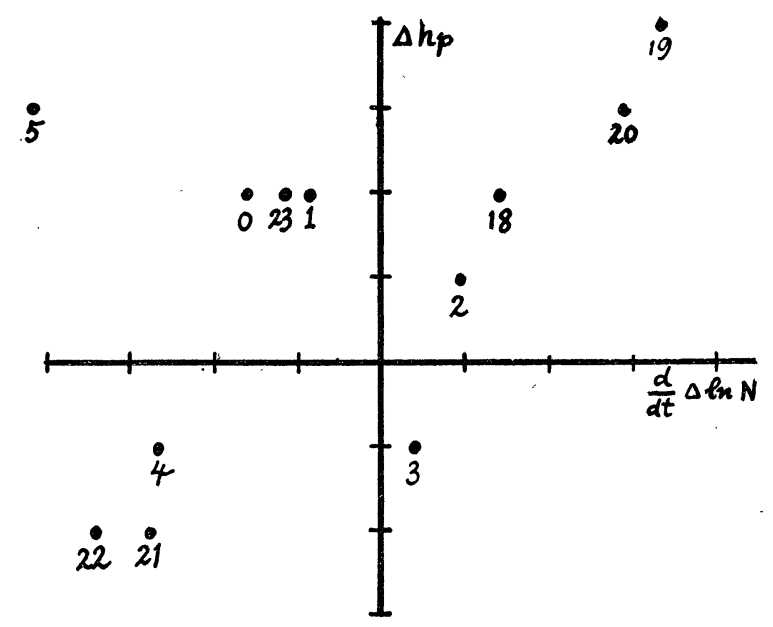

Fig. 1 (a) January, 1949

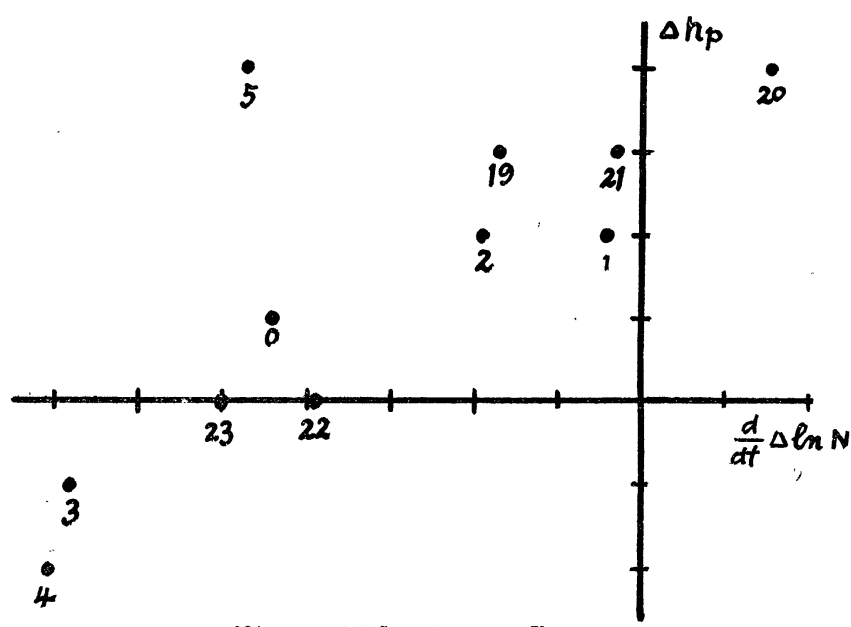

Fig. 1 (b) February, 1949

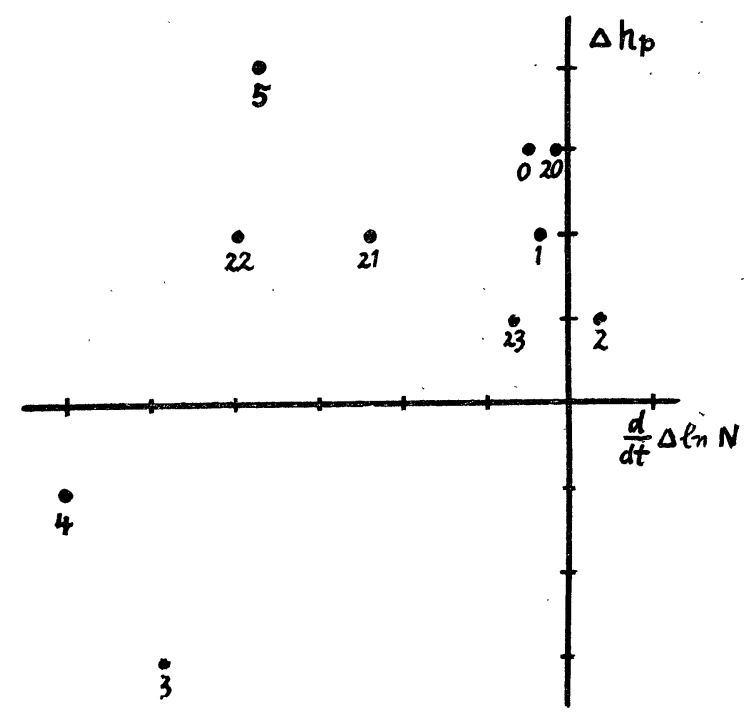

Fig. 1 (c) March, 1949 scales of both axes are arbitrary; the numbers attached to respective points in the figures show the hours of observation (J.S.T.).

We can see from these figures that in winter these points arrange themselves roughly along straight lines, with the exception of points corresponding to hours near sunrise or sunset. This exception is not surprising in view of the fact that we can not obtain accurate values of $\frac{d}{d t} \Delta \ln N$ by numerical differentiation near sunrise or sunset (especially near the former) owing to the rapid change in slope of the diurnal variation curve of electron density. On the other hand, linear relation does not so well hold between $\frac{d}{d t} d$ $\ln N$ and $\Delta h_{p}$ for other seasons. It seems that this is, on the one hand, because the height determination in the ionospheric sounding is subject to not small errors, sometimes as high as $10 \mathrm{~km}$., so that the difference in height between two stations may be, in unfavourable cases, in error by about $20 \mathrm{~km}$., but, on the other, because height

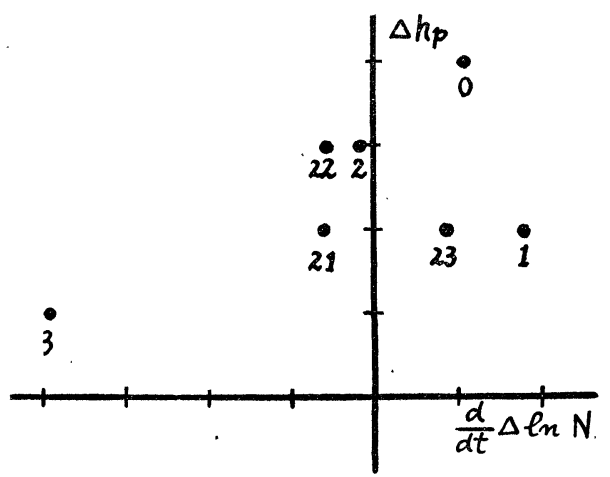

Fig 1 (d) June, 1949 


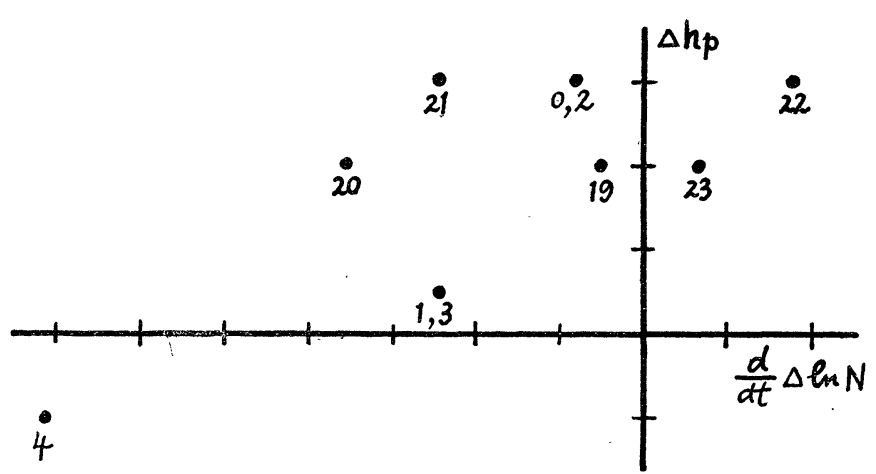

Fig. 1 (e) September, 1949

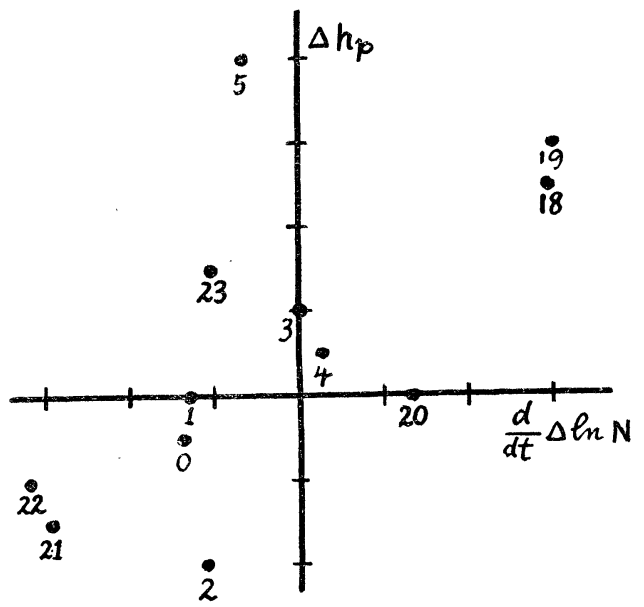

Fig. 1 (f) December, 1949

change of the F2 layer is not very great in seasons other than winter. In view of these disturbing factors it will not be far from truth that the linear relation between $\frac{d}{d t} \Delta \ln N$ and $\Delta h_{p}$ is also existing for seasons other than winter. An evidence for this may be provided by the fact that, if we take a weighted running average of $h_{p}$ instead of $h_{p}$ itself for each hour, the linear relation becomes much more clear as shown in Fig. 2 for June, 1949.

Now, if recombination theory is correct, there must be a linear relation between $\frac{d}{d t} \Delta \ln N$ and $\Delta N$, and the correlation must be negative, as shown in Eq.

(6). So an examination was made of this relation, and an example of its results is shown in Fig. 3 (February, 1949). We can see from the figure that the points are scattering more widely than in the corresponding Fig. 1 (b). But, what is more important, the correlation, if existing at all, is rather positive than negative, which contradicts the expectation from Eq. (6). Thus we can hardly support the recombination theory for electron dissipation during the night.

Entering into more details, we have then fitted Eq. (8) to observational data and determined the most probable values of $\boldsymbol{B}_{0}$ / $H_{2}$ and $B_{0} / H_{1}$. In view of the above described facts, good results will not be expect-

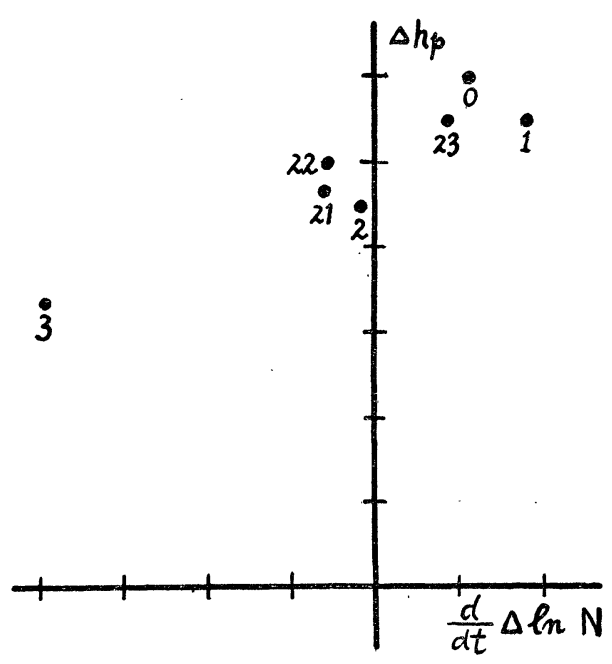

Fig. 2 June, 1949 $\left(\begin{array}{l}\text { Weighted running mean of } \\ \Delta h_{p} \text { is used }\end{array}\right.$ ed except for winter. This was indeed the case. But in no case we have obtained 
very unreasonable values; such as negative ones, for these parameters. The ratio

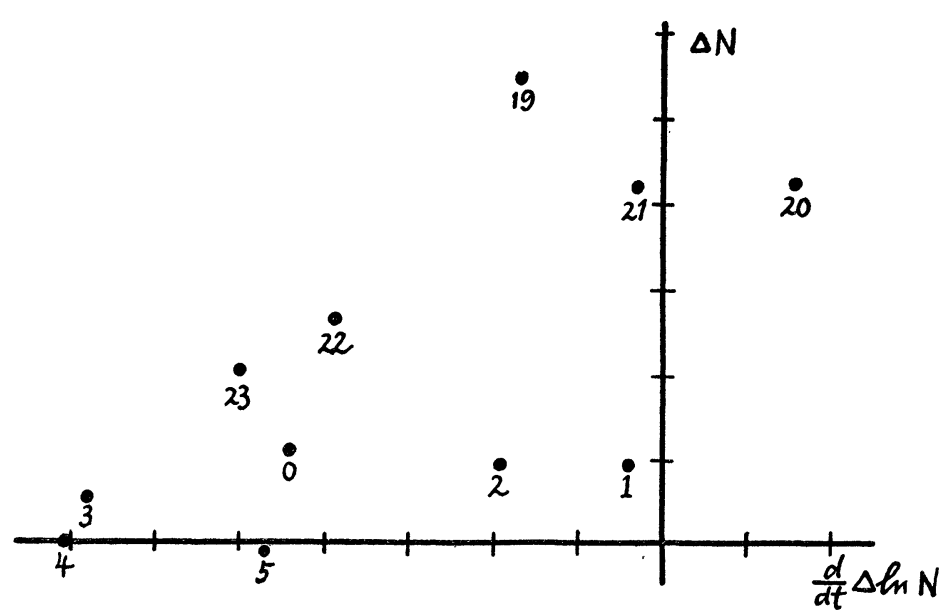

Fig. 3 February, 1949 $H_{2}: H_{1}$ was $1.8,1.6$, and 2.0 for January; February, and December, 1949, respectively. In other seasons also this ratio had a tendency to be larger than 1 , the average of all (including the above mentioned) being 1.9. Though we it can not place much weight on these figures, it is interesting to note that the result of analysis indicates higher upper atmospheric temperatures at Yamagawa $\left(31^{\circ} 12.5^{\prime} \mathrm{N}\right)$ than at Kokubunji $\left(35^{\circ} 42.4^{\prime} \mathrm{N}\right)$, which is consistent with our expectation.

4. Conclusion

Thus the comparison of nocturnal electron density variations at Kokubunji and Yamagawa showed that electrons disappear in the $\mathrm{F} 2$ region according to a linear law rather than a quadratic one, and the temperature of the upper atmosphere is somewhat higher at Yamagawa than at Kokubunji. But, in view of the limit of accuracy of height determination in ionospheric sounding, we can not hope to draw any quantitative deductions from such an analysis, and we must wait until more accurate data become available.

[1] T. Yonezawa, Rep. Ionosphere Res. Japan, 5, 1 (1951) 